\title{
A retrospective analysis of women diagnosed with unclassified HPV genotypes
}

\author{
Can Turkler, Tunay Kiremitli
}

Department of Gynecology and Obstetrics, Faculty of Medicine, Erzincan Binali Yıldırım University, Erzincan, Turkey

Submitted: 9 February 2019

Accepted: 9 April 2019

Arch Med Sci Civil Dis 2019; 4: e22-e27

DOI: https://doi.org/10.5114/amscd.2019.85654

Copyright $\odot 2019$ Termedia \& Banach

\section{Abstract}

Introduction: This study primarily aimed to investigate the associations between unclassified HPV genotypes and cervical lesions.

Material and methods: This was a retrospective review of 411 patients with HPV positivity. The participants were divided into two groups: Group X contained HPV genotypes 16, 18, 31, 33, 35, 39, 45, 51, 52, 56, 58, 59 and 68 while group $Y$ contained unclassified HPV genotypes such as $42,54,61,62$, $71,81,83,84$ and 89 . The $X$ and $Y$ groups were compared in terms of demographic characteristics and related cervical pathologies. We investigated the potential of HPV genotypes in group Y to develop cervical neoplasia.

Results: HPV 16 is the most common genotype in group $X(28.5 \%)$ whereas HPV 83 is the most common genotype in group $Y(4.9 \%)$. Group $X$ and group $Y$ were statistically similar with respect to age $(p=0.231)$, parity ( $p=0.617)$, occupation $(p=0.535)$, marital status $(p=0.644)$, education level $(p=0.316)$, smoking $(p=0.352)$, gynecological findings $(p=0.201)$, Pap smear results $(p=0.427)$, and colposcopy findings $(p=0.476)$. When compared to group X, normal cervical biopsy was significantly more frequent (18.6\% vs. $39.1 \%)$, chronic cervicitis was significantly less frequent $(49.5 \%$ vs. $36.9 \%)$, CIN 1 was significantly less frequent (12.9\% vs. $8.7 \%)$ and CIN 2 was significantly less frequent $(5.6 \%$ vs. $2.2 \%)(p=0.012)$. Cervical cancer was diagnosed in three patients of group $\mathrm{X}$ and one patient of group $\mathrm{Y}(1.6 \%$ vs. $2.2 \%)$.

Conclusions: Screening programs for cervical cancer are generally performed to detect HPV genotypes with high oncogenic potential. The importance of unclassified HPV genotypes should be investigated in large scale studies so that the success of screening programs and vaccination can be improved.

Key words: cervical cancer, colposcopy, human papillomavirus, unclassified.

\section{Introduction}

Human papillomavirus (HPV) is a sexually transmitted virus which is associated with condyloma, cervical neoplasia and carcinoma. The results of HPV transmission depend on the oncogenic potential of the virus and the immunity of the host [1].

Invasive cervical cancer is the second most common malignancy of women living all over the world. HPV infection is the most important risk factor for invasive cervical cancer [2, 3]. HPV genotypes are divided into five groups based on their oncogenic capacity (Table I). Group 1 is the highest potential risk factor for invasive cervical cancer. Groups 2A and

\author{
Corresponding author: \\ Asist. Prof. Can Turkler \\ Department \\ of Gynecology \\ and Obstetrics \\ Faculty of Medicine \\ Erzincan Binali \\ Yıldırım University \\ Erzincan, Turkey \\ E-mail: dr_canturkler@yahoo. \\ com
}


Table I. Distribution of HPV types based on their oncogenic capacity

\begin{tabular}{|lc|}
\hline Group 1 (carcinogenic to humans) & $16,18,31,33,35,39,45,51,52,56,58,59$ \\
\hline Group 2A (probably carcinogenic to humans) & 68 \\
\hline Group 2B (possibly carcinogenic to humans) & $26,30,34,53,66,67,69,70,73,82,85,97$ \\
\hline Group 3 (unclassifiable as to carcinogenicity in humans) & 6,11 \\
\hline Unclassified* & $42,54,61,62,71,81,83,84,89$ \\
\hline
\end{tabular}

*In our study we found 9 unclassified genotypes.

2B include probably and possibly oncogenic HPVs. Group 3 is responsible for benign genital warts but there is not enough information about the role of unclassified HPV genotypes in the carcinogenesis of cervical tumors [4].

The prevalence of HPV genotypes varies around the world. For example, the highest HPV prevalence is about $20-30 \%$ in Africa and Latin Ameri$\mathrm{ca}$, and the lowest HPV prevalence is about 6-7\% in Southern Europe and South East Asia [5]. The HPV genotypes vary with geographic location. For instance, the leading 5 HPV genotypes are HPV16, 53, 52, 18, and 39 for America whereas these genotypes are HPV16, 52, 58, 18, and 56 for Asia [6].

Specifying the distribution of HPV genotypes according to geographic regions is very important for determining an efficient prevention strategy that includes screening programs and vaccination. It is also important to clarify the role of unclassified HPV genotypes in the carcinogenesis of cervical tumors so that HPV vaccines and their efficiency can be improved. This study primarily aimed to investigate the associations between unclassified HPV genotypes and pre-malignant and malignant cervical lesions.

\section{Material and methods}

The present study was conducted in accordance with the approved guidelines and the principles expressed in the Declaration of Helsinki. (Ethics Committee Number: (12.09.2018-30/01)). Written informed consent was obtained from each participant.

There is a cervical cancer screening program conducted by the Ministry of Health in Turkey. The patients diagnosed with HPV positivity by the Cancer Early Diagnosis Screening and Training Center were referred to the study center between January 2014 and December 2018. This is a retrospective review of 411 patients with HPV positivity. The inclusion criteria were being between 30 and 65 years old, being sexually active, having no pregnancy during the study, having no hysterectomy or cervical resection, and not being diagnosed with cervical cancer in the past years. The exclusion criteria were being HIV positive, having vaginal bleeding during pelvic examination, hav- ing multiple HPV infections, using vaginal medications or having sexual intercourse in the last $48 \mathrm{~h}$ before the examination.

The participants were divided into two groups: Group X contained HPV genotypes 16, 18, 31, 33, $35,39,45,51,52,56,58,59$ and 68 while group $Y$ contained unclassified HPV genotypes such as 42 , $54,61,62,71,81,83,84$ and 89 . The $X$ and $Y$ groups were compared in terms of demographic characteristics and related cervical pathologies. The primary outcome of our study is to show the potential of unclassified HPV genotypes in the development of pre-malignant and malignant cervical lesions.

\section{Cervical smear and HPV sampling}

Cervical smears and HPV samples were collected from all participants by a doctor or nurse trained in cervical cancer screening, according to the standard sampling procedure at the Cancer Early Diagnosis Screening and Training Center.

\section{HPV-DNA isolation and genotyping}

Cervical specimens were collected and transported using the HC2 HPV DNA (Qiagen Gaithersburg, Inc. USA) Collection Device. The specimen was kept in $3 \mathrm{ml}$ of sample transport medium. HPV DNA was extracted and amplified by Hybrid Capture-2 and polymerase chain reaction (PCR), using the known protocols in the literature [7-9]. The restriction fragment length polymorphism method was also used to classify the unknown HPV types. HPV genotyping was performed with the DYEnamic ET Terminator Cycle Sequencing Kit (Amersham Biosciences Corp., NJ, USA) and $A B I$ PRISM 310 Genetic Analyzer at Iontek, Turkey.

\section{Colposcopy}

Colposcopy was performed in 224 patients (54.5\%) due to previously established indications by World Health Organization (Table II) [10]. This procedure was carried out by means of a colposcope (LEICA CLS 150 XC, Germany) with a digital optical system (LEICA DFC 295, Germany). A cervical biopsy was taken from suspicious areas such as acetowhite epithelium, atypical vascularization, punctuation, mosaicism or leukoplakia. 
Table II. Indications for colposcopy

\begin{tabular}{|c|}
\hline Suspicious-looking cervix \\
\hline Invasive carcinoma on cytology \\
\hline CIN 2 or CIN 3 on cytology \\
\hline $\begin{array}{l}\text { Persisting (for more than } 12-18 \text { months) low-grade } \\
\text { (CIN 1) abnormalities on cytology }\end{array}$ \\
\hline CIN 1 on cytology \\
\hline Persistently unsatisfactory quality on cytology \\
\hline Infection with oncogenic human papillomaviruses \\
\hline $\begin{array}{l}\text { Acetopositivity on visual inspection with acetic acid } \\
\qquad(\mathrm{VIA})\end{array}$ \\
\hline $\begin{array}{l}\text { Acetopositivity on visual inspection with acetic acid } \\
\text { using magnification (VIAM) }\end{array}$ \\
\hline Positive on visual inspection with Lugol's iodine (VILI) \\
\hline
\end{tabular}

\section{Statistical analysis}

Collected data were analyzed by SPSS version 22.0 (IBM Corp., Armonk, NY, US). Continuous variables were expressed as mean \pm standard deviation (SD) or median (min.-max.), and categorical variables were denoted as numbers or percentages, if applicable. Pearson's $\chi^{2}$ test and the Mann-Whitney $U$ test were used for comparisons. Two-tailed $p$-values $<0.05$ were considered to be statistically significant.

\section{Results}

Table III shows the distribution of HPV genotypes. HPV 16 is the most common genotype in group $X(28.5 \%)$ whereas HPV 83 is the most common genotype in group $Y(4.9 \%)$. Table IV shows the demographic, clinical and pathological characteristics of both groups. Group $X$ and group $Y$ were statistically similar with respect to age $(p=0.231)$, parity $(p=0.617)$, occupation $(p=0.535)$, marital status $(p=0.644)$, education level $(p=0.316)$, smoking $(p=0.352)$, gynecological findings $(p=0.201)$, Pap smear results $(p=0.427)$, and colposcopy findings ( $p=0.476)$. When compared to group $X$, normal cervical biopsy was significantly more frequent $(18.6 \%$ vs. $39.1 \%$ ), chronic cervicitis was significantly less frequent (49.5\% vs. $36.9 \%)$, CIN 1 was significantly less frequent (12.9\% vs. $8.7 \%)$ and $\mathrm{CIN} 2$ was significantly less frequent $(5.6 \%$ vs. $2.2 \%)$ $(p=0.012)$. Cervical cancer was diagnosed in 3 patients of group $X$ and 1 patient of group $Y$. One patient in group $X$ had cervical adenocancer, 2 patients in group $X$ had cervical squamous cell cancer and the patient in group $Y$ had microinvasive squamous cell cancer. The distribution of cervical lesions according to HPV genotypes is displayed in Table $V$ for group $Y$.
Table III. Distribution of HPV genotypes in the study group

\begin{tabular}{|c|c|c|}
\hline HPV genotypes & Frequency & Percent \\
\hline 16 & 117 & 28.5 \\
\hline 51 & 34 & 8.3 \\
\hline 39 & 25 & 6.1 \\
\hline 31 & 24 & 5.8 \\
\hline 18 & 20 & 4.9 \\
\hline 83 & 20 & 4.9 \\
\hline 71 & 19 & 4.6 \\
\hline 45 & 14 & 3.4 \\
\hline 52 & 14 & 3.4 \\
\hline 35 & 13 & 3.2 \\
\hline 89 & 13 & 3.2 \\
\hline 56 & 12 & 2.9 \\
\hline 54 & 11 & 2.7 \\
\hline 58 & 11 & 2.7 \\
\hline 81 & 11 & 2.7 \\
\hline 42 & 10 & 2.4 \\
\hline 62 & 10 & 2.4 \\
\hline 68 & 9 & 2.2 \\
\hline 84 & 9 & 2.2 \\
\hline 61 & 7 & 1.7 \\
\hline 33 & 5 & 1.2 \\
\hline 59 & 3 & 0.7 \\
\hline Total & 411 & 100 \\
\hline
\end{tabular}

\section{Discussion}

The role of unclassified HPV genotypes in the carcinogenesis of cervical tumors has not been fully clarified and the number of studies related to this subject is limited in the literature. Barut et al. found that women who had an abnormally appearing cervix during clinical examination should be evaluated by Pap smear and colposcopy [11]. This study aims to evaluate the role of HPV genotypes in the carcinogenesis of cervical tumors by Pap smear, colposcopy and colposcopy guided biopsy results.

The prevalence of HPV infections in women is strongly influenced by demographic and geographic characteristics [12]. Kulhan et al. previously reported about the prevalence of HPV genotypes in Turkish women. In the present study, the demographic data related to age, occupation, marital status, education level and smoking in this 
Table IV. Demographic, clinical and pathological characteristics

\begin{tabular}{|c|c|c|c|}
\hline Characteristics & Group X $(n=301)(\%)$ & Group Y $(n=110)(\%)$ & $P$-value \\
\hline Age, mean \pm SD [years] & $44.7 \pm 9.4$ & $43.3 \pm 8.5$ & 0.231 \\
\hline Parity: & & & 0.617 \\
\hline Nullipara & $37(12.3)$ & $14(12.7)$ & \\
\hline Multipara & $264(87.7)$ & $96(87.3)$ & \\
\hline Occupation: & & & 0.535 \\
\hline Housewife & $180(59.8)$ & $68(61.8)$ & \\
\hline Worker & $121(40.2)$ & $42(38.2)$ & \\
\hline Marital status: & & & 0.644 \\
\hline Single & $28(9.4)$ & $10(9.1)$ & \\
\hline Married & $241(80)$ & $85(77.3)$ & \\
\hline Widow & $32(10.6)$ & $15(13.6)$ & \\
\hline Education level: & & & 0.316 \\
\hline Illiterate & $36(11.9)$ & $11(10)$ & \\
\hline Primary education & $136(45.2)$ & $48(43.6)$ & \\
\hline High school & $98(32.6)$ & $41(37.3)$ & \\
\hline University & $31(10.3)$ & $10(9.1)$ & \\
\hline Smoking: & & & 0.352 \\
\hline No & $181(60.2)$ & $65(59.1)$ & \\
\hline Yes & $120(39.8)$ & $45(40.9)$ & \\
\hline Gynecological findings: & & & 0.201 \\
\hline Normal & $233(77.4)$ & $86(78.2)$ & \\
\hline Genital warts & $14(4.7)$ & $9(8.1)$ & \\
\hline Post-coital bleeding & $54(17.9)$ & $15(13.7)$ & \\
\hline Pap smear results: & & & 0.427 \\
\hline Normal & $264(87.7)$ & $90(81.8)$ & \\
\hline ASC-US & $19(6.3)$ & $10(9.2)$ & \\
\hline ASC-H & $2(0.7)$ & $1(0.9)$ & \\
\hline LSIL & $16(5.3)$ & $9(8.1)$ & \\
\hline HSIL & 0 & 0 & \\
\hline Colposcopy findings: & & & 0.476 \\
\hline Normal & $213(70.7)$ & $63(57.2)$ & \\
\hline Acetowhite epithelium (ACW) & $22(7.4)$ & $35(31.8)$ & \\
\hline Atypical vascularization (AV) & $18(5.9)$ & $4(3.7)$ & \\
\hline $\mathrm{ACW}+\mathrm{AV}$ & $25(8.4)$ & $5(4.6)$ & \\
\hline Punctuation & $17(5.7)$ & $1(0.9)$ & \\
\hline Mosaicism & $6(1.9)$ & $1(0.9)$ & \\
\hline Leukoplakia & 0 & $1(0.9)$ & \\
\hline Cervix biopsy results: & & & 0.012 \\
\hline Normal & $33(18.6)$ & $18(39.1)$ & \\
\hline Chronic cervicitis & $88(49.5)$ & $17(36.9)$ & \\
\hline CIN1 & $23(12.9)$ & $4(8.7)$ & \\
\hline $\mathrm{CIN} 2$ & $10(5.6)$ & $1(2.2)$ & \\
\hline $\mathrm{CIN} 3$ & $21(11.8)$ & $5(10.9)$ & \\
\hline Cancer & $3(1.6)$ & $1(2.2)$ & \\
\hline Total & 178 & 46 & \\
\hline
\end{tabular}

ASC-US - atypical squamous cells of undetermined significance, ASC-H - atypical squamous cell-cannot exclude high-grade squamous intraepithelial lesion, LSIL - low-grade squamous intraepithelial lesion, HSIL - high-grade squamous intraepithelial lesion, CIN - cervical intraepithelial neoplasia. 
Table V. Distribution of cervical lesions according to HPV genotypes in group Y

\begin{tabular}{|lcccc|}
\hline HPV type & \multicolumn{5}{c|}{ Cervical biopsy results } \\
\cline { 2 - 5 } & CIN 1 & CIN 2 & CIN 3 & Cancer \\
\hline $42(n=10)$ & - & - & - & - \\
\hline $54(n=11)$ & + & - & - & - \\
\hline $61(n=7)$ & + & - & + & - \\
\hline $62(n=10)$ & - & - & ++ & + \\
\hline $71(n=19)$ & + & - & ++ & - \\
\hline $81(n=11)$ & - & - & + & - \\
\hline $83(n=20)$ & + & - & - & - \\
\hline $84(n=9)$ & - & - & - & - \\
\hline $89(n=13)$ & - & - & & - \\
\hline
\end{tabular}

(-): zero, (+): 1 case, $(++)$ : 2 case.

study were similar to those of Kulhan et al., but the number of multiparous patients was lower in this study (78.5\% to $87.5 \%)$ [13]. The most common colposcopy finding was acetowhite epithelium in the study of Kulhan et al., but the most common finding was atypical vascularization in the study of Barut et al. [11]. Colposcopy findings of $X$ and $Y$ groups in our study were different. In the $X$ group, the most common finding was acetowhite epithelium + atypical vascularization, but the most common finding in the $Y$ group was acetowhite epithelium.

Similar to the present study, the most common genotype was HPV 16 in patients diagnosed with cervical cancer [13-15]. Annunziata et al. declared that the most common unclassified genotype was HPV 81, in Italian women [4]. However, in Turkish women included in our study, the most common unclassified genotype was HPV 83.

Annunziata et al. reported that none of the patients in the unclassified HPV group had a high-grade squamous intraepithelial lesion in Pap smear results, in parallel with the results of our study. However, in the literature, it was found that unclassified HPV genotypes can exert carcinogenic effects especially in immunosuppressive and HIV-positive patients $[16,17]$. HIV-positive women were not included in this study, but according to the colposcopy guided biopsy results, there was one case of squamous cell carcinoma case related to HPV 81 in group Y. In this case, smoking was the only risk factor detected.

Baloch et al. identified 6 unclassified HPV genotypes (HPV 42, 55, 61, 71, 81, 83) in their epidemiological study in which a total of 17,898 women were evaluated. In the present study, there was no cancer case in the group of patients with unclassified HPV genotypes; however, the incidence of CIN1, CIN2 and CIN3 was higher in the HPV 81 positive group than the others [18].
It can be speculated that the prevalence of unclassified HPV genotypes and their oncogenic potentials are underestimated because researchers have focused on HPV genotypes with higher oncogenic potential [19-22]. Recently, clinicians discovered that cervical cancer can be prevented by vaccination and studies have accelerated in this direction. However, the distribution of HPV genotypes is not homogeneous and varies with respect to geographic location. Since the role of unclassified HPV genotypes in the carcinogenesis of cervical tumors has not been clarified, more studies should be performed on this subject. In this way, it is possible to improve HPV vaccines and increase their effectiveness.

This study has some limitations. First, it is a retrospective study with a small sample size. Second, the behavioral risk factors, such as condom use, multiple sexual partners and the first sexual intercourse age, have not been considered. The third disadvantage was the lack of long-term data related to the persistence of unclassified HPV genotypes.

In conclusion, the significance of cervical cancer screening programs has become prominent. These screening programs are generally performed to detect HPV genotypes with high oncogenic potential. The importance of unclassified HPV genotypes should be investigated in larger patient series so that the success of screening programs and vaccination can be improved. It should also be taken into account whether there is an additional risk factor such as smoking when deciding on the histopathological examination of women who are diagnosed with unclassified HPV genotypes.

\section{Conflict of interest}

The authors declare no conflict of interest. 


\section{References}

1. Nguyen AQ, Mandigo M, Coleman JS. Cervical cancer screening for women with disabilities: time for a new approach? J Low Genit Tract Dis 2018; 22: 318-9.

2. Beyazit F, Silan F, Gencer M, et al. The prevelance of human papillomavirus (HPV) genotypes detected by PCR in women with normal and abnormal cervico-vaginal cytology. Ginekol Pol 2018; 89: 62-7.

3. Gottwald L, Korczyński J, Góra E, et al. Clear cell adenocarcinoma of the uterine cervix in a 24-year-old woman. Case report and review of the literature. Arch Med Sci 2012; 8: 578-81.

4. Annunziata C, Stellato G, Greggi S, et al. Prevalence of "unclassified" HPV genotypes among women with abnormal cytology. Infect Agent Cancer 2018; 13: 26.

5. Biological agents. Volume 100 B. A review of human carcinogens. IARC Monogr Eval Carcinog Risks Hum 2012, 100: 1-441.

6. Barut MU, Yildirim E, Kahraman M, et al. Human papilloma viruses and their genotype distribution in women with high socioeconomic status in Central Anatolia, Turkey: a pilot study. Med Sci Monit 2018; 24: 58-66.

7. Evans MF, Adamson CS, Papillo JL, St John TL, Leiman G, Cooper K. Distribution of human papillomavirus types in thinprep papanicolaou tests classifed according to the Bethesda 2001 terminology and correlations with patient age and biopsy outcomes. Cancer 2006, 106: 1054-64.

8. Bauer HM, Ting Y, Greer CE, et al. Genital human papillomavirus infection in female university students as determined by a PCR-based method. JAMA 1991; 265: 472-7.

9. Gravitt PE, Peyton CL, Alessi TQ, et al. Improved amplification of genital human papillomaviruses. J Clin Microbiol 2000; 38: 357-61.

10. Sellors JW, Sankaranarayanan R. Colposcopy and treatment of cervical intraepithelial neoplasia: a beginners' manual, Chapter 4: An introduction to colposcopy: indications for colposcopy, instrumentation, principles and documentation of results. http://screening.iarc.fr/ colpochap.php?chap=4\&lang=1 (access: 2018.12.06).

11. Barut MU, Kale A, Kuyumcuoğlu U, et al. Analysis of sensitivity, specificity, and positive and negative predictive values of smear and colposcopy in diagnosis of premalignant and malignant cervical lesions. Med Sci Monit 2015; 21: 3860-7.

12. Clifford GM, Gallus S, Herrero R, et al. Worldwide distribution of human papillomavirus types in cytologically normal women in the International Agency for Research on Cancer HPV prevalence surveys: a pooled analysis. Lancet 2005; 366: 991-8.

13. Kulhan M, Kulhan NG, Seven $\mathrm{Y}$, et al. Estimation of the prevalence and distribution of HPV genotypes and identification of related risk factors among Turkish women. Contemp Oncol (Pozn) 2017; 21: 218-23.

14. Zhang C, Zhang C, Huang J, Wu Z, Mei X, Shi W. Prevalence and genotype distribution of human papillomavirus among females in the suburb of Shanghai, China. J Med Virol 2018; 90: 157-64.

15. Wolday D, Derese M, Gebressellassie S, et al. HPV genotype distribution among women with normal and abnormal cervical cytology presenting in a tertiary gynecology referral Clinic in Ethiopia. Infect Agent Cancer 2018; 13: 28.

16. Tornesello ML, Duraturo ML, Giorgi-Rossi P, et al. Human papillomavirus (HPV) genotypes and HPV16 variants in human immunodeficiency virus-positive Italian women. J Gen Virol 2008; 89: 1380-9.
17. Garbuglia AR, Piselli P, Lapa D, et al. Frequency and multiplicity of human papillomavirus infection in HIV-1 positive women in Italy. J Clin Virol 2012; 54: 141-6.

18. Baloch Z, Li Y, Yuan T, et al. Epidemiologic characterization of human papillomavirus (HPV) infection in various regions of Yunnan Province of China. BMC Infect Dis 2016; 16: 228.

19. Tornesello ML, Losito S, Benincasa G, et al. Human papillomavirus (HPV) genotypes and HPV16 variants and risk of adenocarcinoma and squamous cell carcinoma of the cervix. Gynecol Oncol 2011; 121: 32-42.

20. Clifford GM, Goncalves MA, Franceschi S. Human papillomavirus types among women infected with HIV: a meta-analysis. AIDS 2006; 20: 2337-44.

21. Clifford GM, Tully S, Franceschi S. Carcinogenicity of human papillomavirus (HPV) types in HIV-positive women: a meta-analysis from HPV infection to cervical cancer. Clin Infect Dis 2017; 64: 1228-35.

22. Ibragimova M, Tsyganov M, Shpileva O, et al. HPV status and its genomic integration affect survival of patients with cervical cancer. Neoplasma 2018; 65: 441-8. 